\title{
Asymptotic regime in $N$ random interacting species
}

\author{
A.Fiasconaro*, D.Valenti and B.Spagnolo \\ Dipartimento di Fisica e Tecnologie Relative, Università di Palermo \\ and INFM-CNR, Group of Interdisciplinary Physics ${ }^{\dagger}$, \\ Viale delle Scienze, I-90128 Palermo, Italy
}

(Dated: September 30, 2005)

\begin{abstract}
The asymptotic regime of a complex ecosystem with $N$ random interacting species and in the presence of an external multiplicative noise is analyzed. We find the role of the external noise on the long time probability distribution of the $i^{t h}$ density species, the extinction of species and the local field acting on the $i^{\text {th }}$ population. We analyze in detail the transient dynamics of this field and the cavity field, which is the field acting on the $i^{\text {th }}$ species when this is absent. We find that the presence or the absence of some population give different asymptotic distributions of these fields.
\end{abstract}

PACS numbers: 05.40.-a, 05.45.-a, 87.23.Cc, 89.75.-k

Keywords: Statistical Mechanics, Population Dynamics, Noise-induced effects, Complex Systems

* e-mail: afiasconaro@gip.dft.unipa.it

$\dagger$ Electronic address: http://gip.dft.unipa.it 


\section{INTRODUCTION}

In recent years great attention has been devoted to population dynamics modelled by generalized Lotka-Volterra systems [1]. Ecosystems are a classic example of complex systems, which became object of study as well by biologists as by physicists [2, 3]. Tools developed in the context of nonequilibrium statistical physics to analyze nonequilibrium nonlinear physical systems provide new insights and at the same time new approaches to the comprehension of the properties of biological and many body systems. A key aspect to understand the complex behavior of ecosystems is the role of the external noise on the dynamics of such systems. Noise-induced effects in population dynamics, such as pattern formation [4, 5], stochastic resonance, noise delayed extinction, quasi periodic oscillations etc... have been investigated with increasing interest [6-10]. The dynamical behavior of ecological systems of interacting species evolves towards the equilibrium states through the slow process of nonlinear relaxation, which is strongly dependent on the random interaction between the species, the initial conditions and the random interaction with environment. The mathematical model here used to analyze the dynamics of $N$ biological species with spatially homogeneous densities is the generalized Lotka-Volterra system with a MalthusVerhulst modelling of the self regulation mechanism and with the addition of an external multiplicative noise source $[11,12]$. We obtain the asymptotic behaviors of the probability distribution of the populations for different values of external noise intensity. To analyze the role of the external noise on the transient dynamics of the species we focus on the long time distribution of the local field, that is the interaction term in the dynamical equation of the $i^{\text {th }}$ species that takes the influence of all other species into account. We find that the presence or the absence of some population give different asymptotic distributions of the local field and of the cavity field (field acting on the $i^{\text {th }}$ species when this is absent) in the absence of external noise. When the noise is switched on the asymptotic local and cavity fields tend to overlap and approximately superimpose each other for very high noise intensity. Finally the long time evolution of the average number of the extinct species is reported for different values of the multiplicative noise intensity. 


\section{THE MODEL}

The dynamical evolution of our ecosystem composed by $N$ interacting species in a noisy environment (climate, disease, etc...) is described by the following generalized Lotka-Volterra equations with a multiplicative noise, in the framework of Ito stochastic calculus

$$
d n_{i}(t)=\left[\left(g_{i}\left(n_{i}(t)\right)+\sum_{j \neq i} J_{i j} n_{j}(t)\right) d t+\sqrt{\epsilon} d w_{i}\right] n_{i}(t), \quad i=1, \ldots, N
$$

where $n_{i}(t) \geq 0$ is the population density of the $i^{t h}$ species at time $t$ and the function $g_{i}\left(n_{i}(t)\right)$

$$
g_{i}\left(n_{i}(t)\right)=\left(\alpha+\frac{\epsilon}{2}\right)-n_{i}(t)
$$

describes the development of the $i^{t h}$ species without interacting with other species. In Eq. (1), $\alpha$ is the growth parameter, the interaction matrix $J_{i j}$ models the interaction between different species $(i \neq j)$, and $w_{i}$ is the Wiener process whose increment $d w_{i}$ satisfy the usual statistical properties $\left\langle d w_{i}(t)\right\rangle=0$, and $\left\langle d w_{i}(t) d w_{j}\left(t^{\prime}\right)\right\rangle=\delta_{i j} \delta\left(t-t^{\prime}\right) d t$. We consider an asymmetric interaction matrix $J_{i j}$, whose elements are randomly distributed according to a Gaussian distribution with $\left\langle J_{i j}\right\rangle=0,\left\langle J_{i j} J_{j i}\right\rangle=0$, and $\sigma_{j}^{2}=J^{2} / N$. Therefore our ecosystem contains $50 \%$ of prey-predator interactions $\left(J_{i j}<0\right.$ and $\left.J_{j i}>0\right), 25 \%$ competitive interactions $\left(J_{i j}>0\right.$ and $\left.J_{j i}>0\right)$, and $25 \%$ symbiotic interactions $\left(J_{i j}<0\right.$ and $\left.J_{j i}<0\right)$. We consider all species equivalent so that the characteristic parameters of the ecosystem are independent of the species. The formal solution of Eq. (1) is

$$
n_{i}(t)=\frac{n_{i}(0) z_{i}(t)}{1+n_{i}(0) \int_{0}^{t} d t^{\prime} z_{i}\left(t^{\prime}\right)}
$$

where

$$
z_{i}(t)=\exp \left[\alpha t+\sqrt{\epsilon} w_{i}(t)+\int_{0}^{t} d t^{\prime} h_{i, l o c}\left(t^{\prime}\right)\right] .
$$

The term $h_{i, l o c}(t)=\sum_{j \neq i} J_{i j} n_{j}(t)$ is the local field acting on the $i^{t h}$ population and represents the influence of other species on the differential growth rate. We note that the dynamical behavior of the $i^{\text {th }}$ population depends on the time integral of the process $z_{i}(t)$ and the time integral of the local field.

In the absence of external noise $(\epsilon=0)$, for a large number of interacting species we can assume that the local field $h_{i}(t)$ is Gaussian with zero mean and variance 


$$
\sigma_{h_{i}}^{2}=\Sigma_{j, k}<J_{i j} J_{i k}><n_{j} n_{k}>=J^{2}\left\langle n_{i}^{2}\right\rangle, \text { with } \quad<J_{i j} J_{i k}>=\delta_{j k} \frac{J^{2}}{N} .
$$

As a consequence, in the absence of external noise, from the fixed-point equation $n_{i}(\alpha-$ $\left.n_{i}+h_{i}\right)=0$, the stationary probability distribution of the populations is the sum of a truncated Gaussian distribution at $n_{i}=0\left(n_{i}>0\right.$ always $)$ and a delta function for extinct species. The initial values of the populations $n_{i}(0)$ have also Gaussian distribution with mean value $\left\langle n_{i}(0)\right\rangle=1$, and variance $\sigma_{n(0)}^{2}=0.03$. The interaction strength $J$ determines two different dynamical behaviors of the ecosystem. Above a critical value $J_{c}=1.1$, the system is unstable and at least one of the populations diverges. Below $J_{c}$ the system is stable and asymptotically reaches an equilibrium state. The equilibrium values of the populations depend both on their initial values and on the interaction matrix. If we consider a quenched random interaction matrix, the ecosystem has a great number of equilibrium configurations, each one with its attraction basin. For an interaction strength $J=1$ and an intrinsic growth parameter $\alpha=1$ we obtain: $\left\langle n_{i}\right\rangle=1.4387,\left\langle n_{i}^{2}\right\rangle=4.514$, and $\sigma_{n_{i}}^{2}=2.44$. These values agree with that obtained from numerical simulation of Eq. (1).

In the presence of external noise $(\epsilon \neq 0)$ we calculate long time probability distribution for different values of the noise intensity. These are shown in the following Fig. 1. For increasing external noise intensity we obtain a larger probability distribution with a lower maximum (see the different scales in Figs. 1 for different noise intensity values). The distribution becomes asymmetric for $\epsilon=0.1$ and tends to become a truncated delta function around the zero value $\left(P\left(n_{i}\right)=\delta\left(n_{i}\right)\right.$ for $n_{i}>0$, and $P\left(n_{i}\right)=0$ for $\left.n_{i} \leq 0\right)$, for further increasing noise intensity. The role of the multiplicative noise is to act as an absorbing barrier at $n_{i}=0[11]$. To confirm this picture we calculate the time evolution of the average number of extinct species for different noise intensities. This time behavior is shown in Fig. 2. We see that this number increases with noise intensity, and after the value $\epsilon=0.1$ reaches quickly the normalized maximum value at $\epsilon=10$.

To analyze in more detail the influence of each species on the long time dynamics of the ecosystem we calculate in this regime the local field $h_{i, l o c}(t)$ and the cavity field $h_{i, c a v}(t)$, which is the field acting on the $i^{t h}$ population when this population is absent. The probability distributions for both local and cavity fields have been obtained by simulations for different species in the presence and in absence of external noise. The results are shown in the next section (see Fig. 3). We found that the probability distributions of the cavity fields differ 
Probability of the Density Species: $\mathrm{P}\left(\mathrm{n}_{\mathrm{i}}\right)$
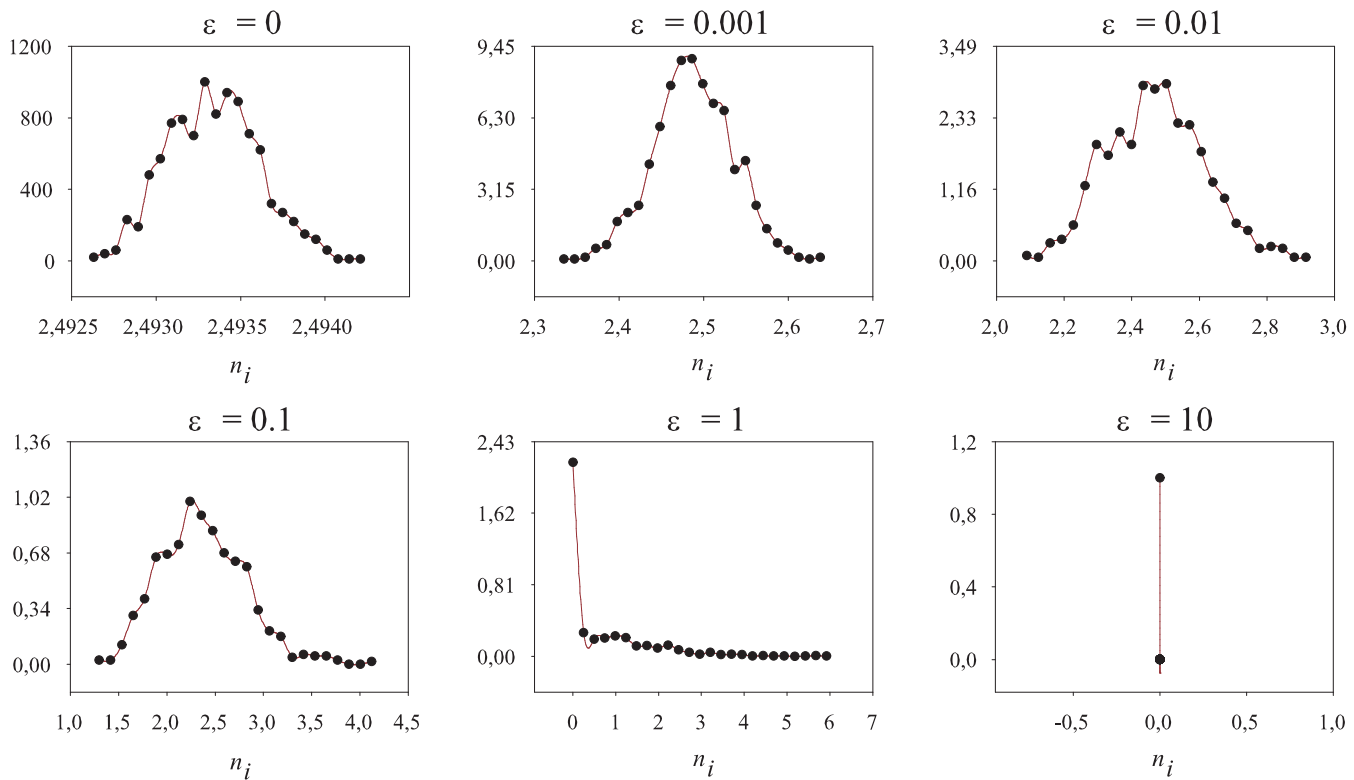

FIG. 1: Probability distribution for the species densities. The values of the external noise intensity are $\epsilon=0,0.001,0.01,0.1,1,10$.

Number of Extinct Species: $\mathrm{N}_{\text {ext }}(\mathrm{t})$
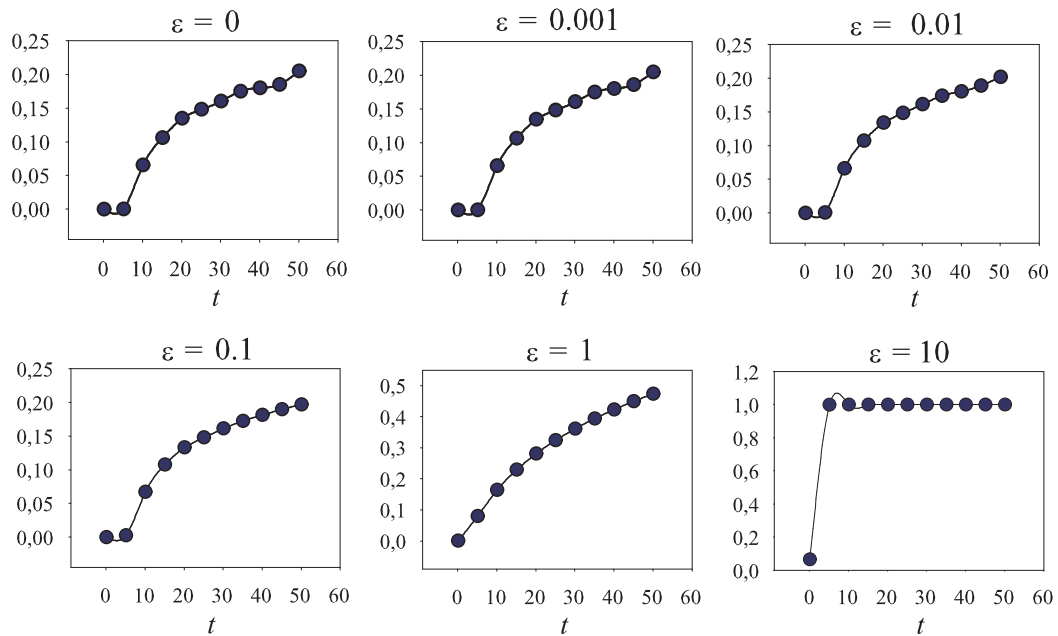

FIG. 2: Time evolution of the average number of extinct species for different noise intensities.

substantially from those of local fields for the same species, while in the presence of noise the two fields overlap. To quantify this overlap between the probabilities distributions of the two fields we define an overlap coefficient $\lambda(t)$, which is the distance between the average values of the two distributions, normalized to their widths 


$$
\lambda(t)=\frac{\bar{h}_{i, l o c}-\bar{h}_{i, c a v}}{\sqrt{\sigma_{i, l o c}^{2}+\sigma_{i, c a v}^{2}}}=\frac{d_{h}(t)}{\sigma_{d}(t)},
$$

where

$$
d_{h}(t)=\bar{h}_{i, l o c}-\bar{h}_{i, c a v}, \quad \sigma_{d}^{2}(t)=\sigma_{i, l o c}^{2}(t)+\sigma_{i, c a v}^{2}(t)
$$

With this definition the distributions start to overlap significantly for $|\lambda| \lesssim 1$, and become strongly overlapping for $|\lambda| \ll 1$.

\section{RESULTS AND COMMENTS}

In the calculation the following parameters have been used: $\alpha=1.2, J=1, \sigma_{J}^{2}=$ 0.005, $N=200$; the number of averaging experiment used is $N_{\exp }=1000$. Concerning the initial condition the parameters are: $\left\langle n_{i}\right\rangle=1, \sigma_{n_{o}}^{2}=0.03$. The dynamics of various species are different even if they are equivalent according to the parameters in the dynamical Eq. (1). However we note that to change the species index by fixing the random matrix or to change the random matrix by fixing the species index is equivalent as regards the asymptotic dynamical regime. Fig. 3 shows for various noise intensities the local and cavity fields Fields Probability Distributions $\mathrm{P}\left(\mathrm{h}_{\mathrm{i}}\right)$
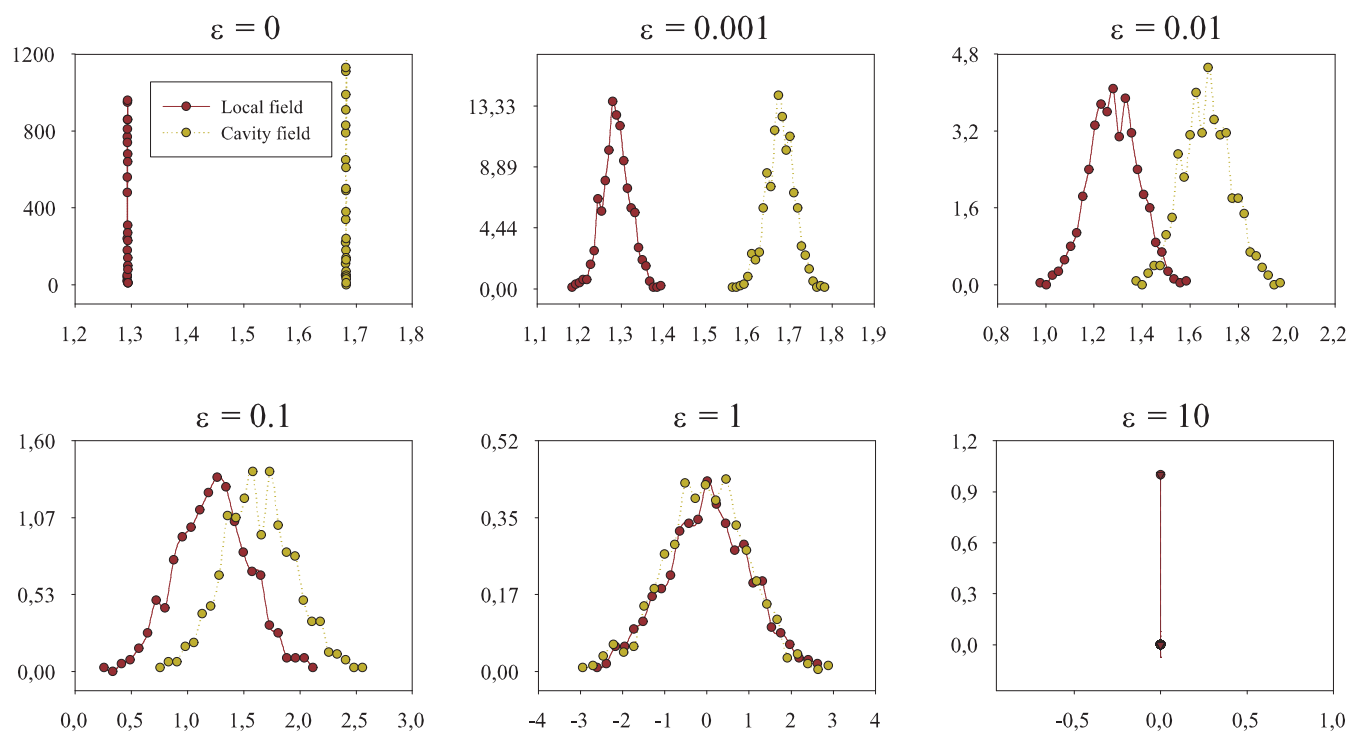

FIG. 3: Probability distribution of both the local (black circles)vand the cavity (white circles) fields for various values of noise intensity $\epsilon=0,0.001,0.01,0.1,1$. The graph are taken at the time $t=50$ unit steps. 
probability distributions at time $t=50$ (a. u.). For noiseless dynamics the distributions of the fields for the species $i=3$ appear very narrow around their mean values and very spaced each other. By increasing the noise intensity, we observe an equal enlargement of the two distributions, maintaining however the same mean values. At $\epsilon=0.1$ the two distributions start to overlap until, for stronger noise intensity $(\epsilon=1)$, they superimpose each other. The overlap coefficient $\lambda(t)$ is equal to zero. The noise makes equivalent all the species in the asymptotic regime and the absence of some species doesn't contribute to any changes in the dynamics of all other species. The last plot in Fig. $3(\epsilon=10)$ gives a delta distribution around zero. This means that, at the time considered in our simulation $(t=50)$ and for this noise intensity, all the species are extinct (see also Fig. 2).
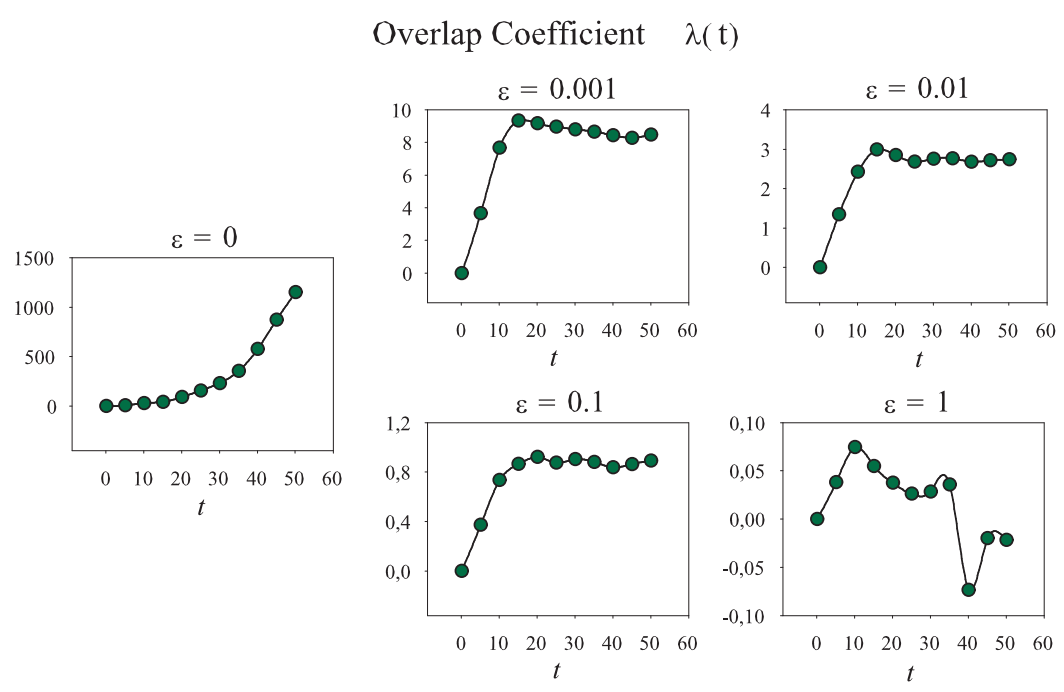

FIG. 4: Time evolution of the overlap coefficient $\lambda(t)$ between the distributions of local and cavity fields for different noise intensities. The increasing behavior of the overlap coefficient as a function of time, in the noiseless case, is due to the narrowing of the two fields distributions $\left(\sigma_{l o c}, \sigma_{c a v} \rightarrow 0\right)$ towards a $\delta$-function. For a noisy environment the distributions tend to enlarge, decreasing the value of the overlap coefficient $\lambda(t)$.

The detailed time evolution of the overlap of the two distributions can be seen from Fig. 4, where it is plotted the coefficient defined in Eq. (6). For $\epsilon=0$ the $\lambda(t)$ coefficient increases with time. This is due to the different time behavior of the distance between the mean values of the field distributions and of their standard deviation $\sigma_{i, l o c}^{2}$ and $\sigma_{i, c a v}^{2}$. The distance $d_{h}(t)=\bar{h}_{i, l o c}-\bar{h}_{i, c a v}$ is almost constant in time, except a rapid initial transient (see Fig. 5, $\epsilon=0$ ), but the corresponding evolution of the distribution widths decreases 
rapidly in time. This effect is due to the quenched random matrix. This behavior remains unchanged until the noise intensity reaches the value of $\epsilon=0.01$. From this value of external noise intensity some differences start to be visible (see Figs. 4, 5 and 6), and at $\epsilon=1$, after some fluctuations, both the distance $d_{h}(t)$ and the overlap coefficient $\lambda(t)$ reach a value close to zero. The two field distributions are totally overlapped.

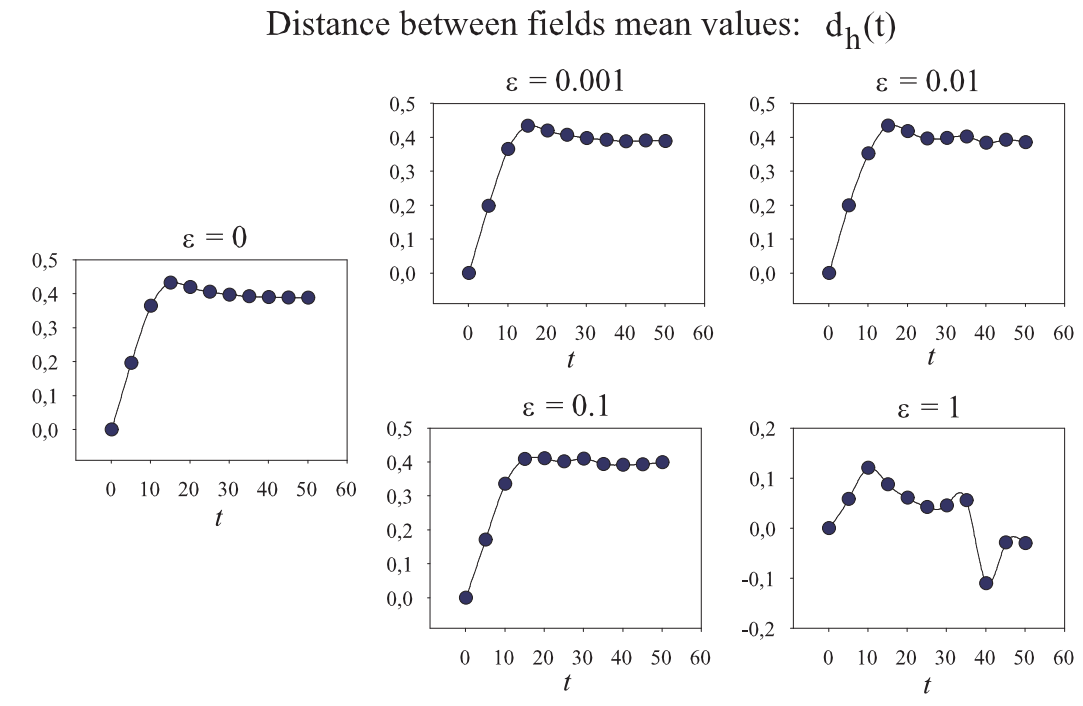

FIG. 5: Time evolution of the distance $d_{h}(t)$ between the mean values of the fields distributions for different noise intensities, namely $\epsilon=0,0.001,0.01,0.1,1$.

Standard Deviation of the Fields: $\sigma_{i}(t)$

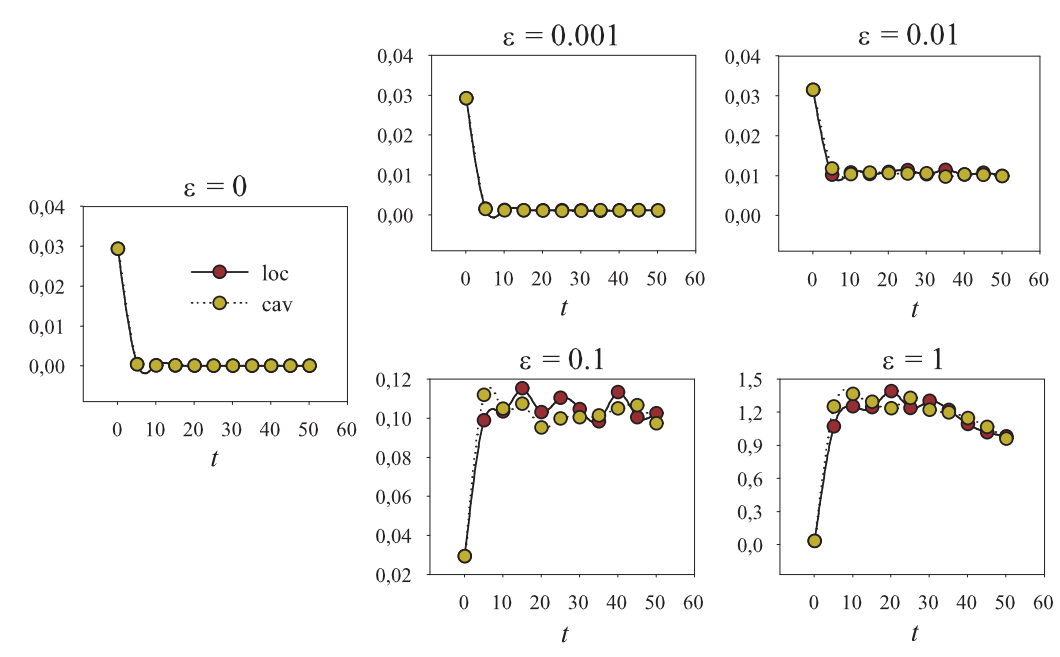

FIG. 6: Time evolution of the standard deviation $\sigma_{i, l o c}^{2}$ (grey circles)and $\sigma_{i, c a v}^{2}$ (black circles) of the field distributions for the same different noise intensities of Fig. 5. 
It is worthwhile to note that the behaviors shown in Figs. 3, 4, 5 and 6 have been found for some species, and changing the species different evolutions of the distribution dynamics appear and in particular of the $\lambda(t)$ coefficient. This is due to complexity of our ecosystem and to the extinction process during the transient dynamics. Moreover this strange behavior, found for some populations and in the asymptotic regime, is reminiscent of the phasetransition phenomenon, and it is related to the following peculiarities of our dynamical system: (i) all the populations are positive; (ii) different initial conditions drive the ecosystem into different attraction basins; and (iii) the complex structure of the attraction basins. While in the presence of noise all the populations seem to be equivalent in the long time regime, some populations, in the absence of external noise, have an asymptotical dynamical behavior such that they significantly influence the dynamics of other species. A more detailed analysis on these points will be done in a forthcoming paper.

\section{CONCLUSIONS}

We analyzed the asymptotic regime of an ecosystem composed by $N$ interacting species in the presence of multiplicative noise. We find the role of the noise on the asymptotic probability distribution of populations and on the extinction process. Concerning the local and the cavity fields, a phase transition like phenomenon is observed. Their probability distributions tend to overlap each other in the presence of external noise, reaching strong overlap for high noise intensity $(|\lambda(t)| \approx 0)$, while they are separated $(|\lambda(t)|>1)$ in the absence of noise. This phenomenon can be ascribed to the complexity of our ecosystem.

\section{Acknowledgements}

This work was supported by MIUR and INFM-CNR.

[1] G. Baxter, A. J. McKane, and M. B. Tarlie, Phys Rev. E 71, 011106 (2005); A. J. McKane and T. J. Newman, Phys. Rev. Lett. 94, 218102 (2005); A. Shabunin, A. Efimov, G.A. Tsekouras, et al., Physica A 347, 117-136 (2005); K. Tokita, Phys. Rev. Lett. 93, 178102 (2004); G. J. Ackland and I. D. Gallagher, Phys. Rev. Lett. 93, 158701 (2004); R. Mankin, A. Sauga, A. 
Ainsaar, et al., Phys. Rev. E 69, 061106 (2004); Y. De Decker, G.A. Tsekouras, A. Provata, et al., Phys. Rev. E 69, 036203 (2004); G.A. Tsekouras, A. Provata, C. Tsallis, Phys. Rev. E 69, 016120 (2004); A. Provata, G.A. Tsekouras, F. Diakonos, et al., Fluct. Noise Lett. 3, L241-L250 (2003).

[2] J. D. Murray, Mathematical Biology, 2nd ed. (Springer, New York, 1993) 54 (1996).

[3] C. Escudero, J. Buceta, F. J. de la Rubia, and Katja Lindenberg, Phys. Rev. E 69, 021908 (2004); T. J. Kawecki and R. D. Holt, Am. Nat. 160, 333 (2002); Michel Droz and Andrzej Pȩkalski, Phys. Rev. E 69, 051912 (2004); I. Giardina, J. P. Bouchaud, M. Mezard, J. Phys. A: Math. Gen. 34, L245 (2001); H Rieger, J. Phys. A: Math. Gen. 22, 3447 (1989).

[4] J. E. S. Socolor, S. Richards, and W. G. Wilson, Phys. Rev. E 63, 041908 (2001).

[5] A. Fiasconaro, D. Valenti and B. Spagnolo, Acta Phys. Pol. B 35, 1491 (2004); D. Valenti, A. Fiasconaro and B. Spagnolo, Acta Phys. Pol. B 35, 1481 (2004); A. La Barbera and B. Spagnolo, Physica A 314, 120 (2001).

[6] B. Spagnolo A. Fiasconaro and D. Valenti, Fluct. Noise Lett. 3, L177 (2003); B. Spagnolo and A. La Barbera, Physica A 315, 201 (2002); A. F. Rozenfeld Rozenfeld, C.J. Tessone, E. Albano, H.S. Wio, Phys. Lett. A 280, 45 (2001); J. M. G. Vilar and R. V. Solé, Phys. Rev. Lett. 80, 4099 (1998).

[7] See the special section on "Complex Systems", Science 284, 79-107 (1999); the special section on "Ecology through Time", Science 293, 623-657 (2001).

[8] D. Valenti, A. Fiasconaro and B. Spagnolo, Physica A 331, 477 (2004).

[9] B. Spagnolo D. Valenti, A. Fiasconaro, Math. Biosciences and Eng. 1, 185 (2004).

[10] D. F. Russel, L. AQ. Wilkens and F. Moss, Nature 402, 291 (2000).

[11] S. Ciuchi, F. de Pasquale and B. Spagnolo, Phys. Rev. E 54, 706 (1996); ibid. 47, 3915 (1993); P. Barrera, S. Ciuchi and B. Spagnolo, J. Phys. A: Math. Gen. 26, L559-L565 (1993).

[12] B. Spagnolo, M. A. Cirone, A. La Barbera and F. de Pasquale, J. Phys.: Condens. Matter 14, 2247 (2002); M. A. Cirone, F. de Pasquale and B. Spagnolo, Fractals 11, 217 (2003); B. Spagnolo, D. Valenti and A. Fiasconaro, Prog. Theor. Phys. Suppl. 157, 312-316 (2005). 